\title{
Inverse correlation between levels of glycated haemoglobin and expression levels of SERCA protein in Mexican patients with type 2 diabetes mellitus
}

\author{
Octavio Galindo-Hernandez ${ }^{1}$, Jesus Rene Machado-Contreras ${ }^{1}$, Rodrigo Martinez-Corella ${ }^{1}$, \\ Tatiana Romero-Garcia², J. Gustavo Vazquez-Jimenez ${ }^{1}$
}

\begin{abstract}
${ }^{1}$ Facultad de Medicina Mexicali, Universidad Autonoma de Baja California, Mexico ${ }^{2}$ Departamento de Bioquímica, Cinvestav-IPN, Mexico
\end{abstract}

Submitted: 3 May 2018

Accepted: 19 August 2018

Arch Med Sci 2020; 16 (5): 1226-1228

DOI: https://doi.org/10.5114/aoms.2020.97970

Copyright (c) 2020 Termedia \& Banach

\begin{abstract}
We examined the association between sarco/endoplasmic reticulum calcium ATPase (SERCA) expression and glycated hemoglobin $\left(\mathrm{HbA}_{1 c}\right)$ levels since alterations in this protein expression are associated with the genesis of insulin resistance. $\mathrm{HbA} 1 \mathrm{c}$ levels and SERCA protein expression from platelets of Mexican patients diagnosed with type 2 diabetes mellitus (T2DM) were analyzed showing lower values of SERCA expression against the normal values we find in healthy people. Interestingly, as diabetes condition got worse; SERCA protein expression decreased gradually until it was undetectable. The results showed an inverse correlation between $\mathrm{HbA}_{1 c}$ and SERCA protein expression in T2DM patients. .
\end{abstract}

Key words: type 2 diabetes mellitus, glycated hemoglobin, SERCA.

Insulin resistance is a systemic condition in which the cell response to this hormone decreases, thus increasing blood glucose levels and inducing the onset of type 2 diabetes mellitus (T2DM), which represents a serious public health issue in Mexico [1]. At the molecular level, there are solid data demonstrating that endoplasmic reticulum stress (ERS) is one of the main causes of insulin resistance genesis [2, 3]. Recent evidence proves that the reduction in SERCA expression induces ERS and the consequent state of insulin resistance [4]. In congruence, overexpression of SERCA protein has been shown to decrease the insulin resistance state in cell cultures and in rodents $[4,5]$. All the features previously mentioned could place SERCA as a possible therapeutic target because rosiglitazone reduces insulin resistance while increasing SERCA expression levels [5, 6].

The study protocol received previous approval from the Hospital General 5 de Diciembre of ISSSTE Mexicali, Mexico (Circular letter number 0985/2017), and was carried out in accordance with the principles of the Declaration of Helsinki, as revised in 2000. T2DM patients and apparently healthy people were recruited after a check-up with their Familiar Physician, obtaining informed written consent to carry out a pilot study.

The study included 27 T2DM diagnosed patients by ADA 2017 Standards of medical care in DM fasting plasma glucose (FPG) $\geq 126 \mathrm{mg} / \mathrm{dl}$ $(7.0 \mathrm{mmol} / \mathrm{l})$, glycated hemoglobin $\left(\mathrm{HbA}_{1 \mathrm{c}}\right) \geq 6.5 \%$, or patients with ran-

\author{
Corresponding author: \\ J. Gustavo Vazquez-Jimenez PhD \\ Facultad de Medicina Mexicali \\ Universidad Autonoma \\ de Baja California \\ Dr. Humberto Torres Sanginés \\ $\mathrm{S} / \mathrm{N}$, Centro Cívico \\ 21000 Mexicali, Mexico \\ E-mail: gustavo.vazquez@ \\ uabc.edu.mx
}


dom plasma glucose $\geq 200 \mathrm{mg} / \mathrm{dl}(11.1 \mathrm{mmol} / \mathrm{l})$ with classic symptoms of hyperglycaemia. The control group included eight apparently healthy individuals (FPG $<100 \mathrm{mg} / \mathrm{dl}, \mathrm{HbA}_{1 \mathrm{c}}<5.7 \%$, body mass index $(\mathrm{BMI})<25 \%$, no comorbidities or pathologic signs or symptoms) of both genders, aged $19-60$ years.

Congestive heart failure, acute or chronic renal failure, type 1 diabetes mellitus, acute or chronic pancreatitis, acute or chronic liver disease, and pregnancy were exclusion criteria.

Glucose and $\mathrm{HbA}_{1 \mathrm{c}}$ levels were determined by standard methods (HITACHI Cobas 600 and Cobas b 101 from Roche, respectively).

Ten millilitres of peripheral blood were drawn into polypropylene tubes containing sodium citrate (Vacutainer System, BD Biosciences). Fractions enriched in platelets were obtained as described previously, with some modifications [7]. Plasma was carefully aspirated from the pellet and centrifuged at $800 \mathrm{~g}$ for $15 \mathrm{~min}$ to obtain plasma fractions enriched in platelets. Finally, fractions enriched in platelets were analysed by western blot.

The protein level of each sample was determined by the micro Bradford protein assay [8].
Proteins (35 $\mu$ g of protein/sample) were separated by SDS-PAGE using 10\% separating gels followed by transfer to polyvinylidene fluoride (PVDF) membranes. Blots were incubated overnight at $4^{\circ} \mathrm{C}$ with primary antibodies (SERCA2 and $\beta$-actin, Santa Cruz Biotechnology) and washed three times with TBS-T buffer before incubation with horseradish peroxidase-conjugated secondary antibodies for $1 \mathrm{~h}$ at $22^{\circ} \mathrm{C}$. Blots were then visualised with Millipore Immobilon western HRP substrate peroxide solution. Quantification of immunoblot films was carried out with ImageJ software.

Average intensities from Western blot films were analysed by two-tailed unpaired Student's $t$-test between two groups, and by One-way ANOVA with Dunnett's post-test for multiple comparison using PRISM, version 7.0 (GraphPad Software, San Diego, (A, USA). A $p$-value $<0.05$ was considered to be statistically significant. Values were reported as mean $\pm \mathrm{SEM}$, and the figures show representative blots.

Initially, in order to corroborate the effectiveness of our antibody to recognise the SERCA protein of human platelets, we processed a rat heart homogenate as a positive control (this antibody recognises the SERCA2 isoform in humans and rats), since

A
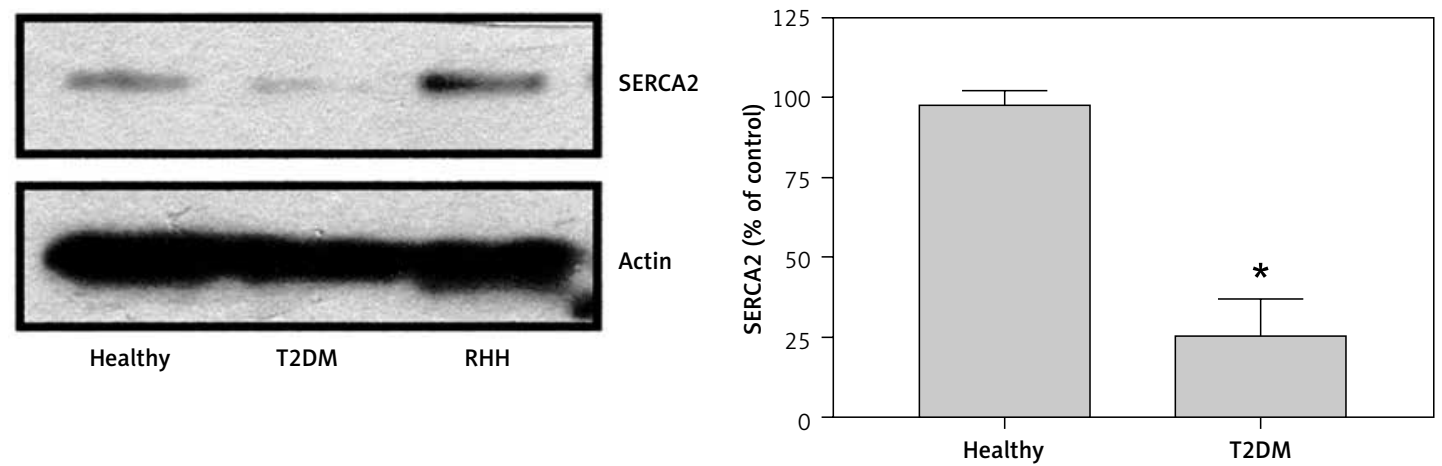

B
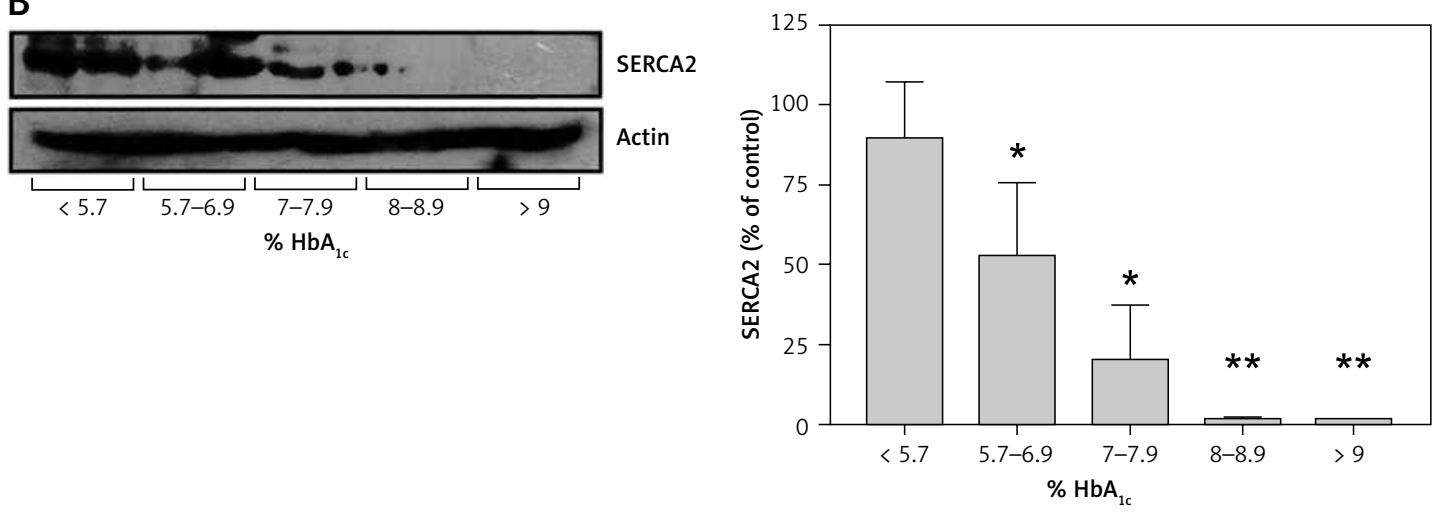

Figure 1. An increase in percentage of glycosylated $\mathrm{HbA}_{1 \mathrm{c}}$ is negatively correlated with SERCA2 protein expression levels in platelets from T2DM patients. A - Fractions enriched in platelets from blood samples of healthy individuals $(n=3)$ and T2DM patients $(n=3)$ compared to rat heart homogenates $(\mathrm{RHH})$. $\mathrm{B}$ - Fractions enriched in platelets from blood samples of healthy individuals $(<5.7, n=8)$, and T2DM patients with increasing percentages of HbA $(5.7-6.9 \%, n=8 ; 7-7.9 \%, n=8 ; 8-8.9 \%, n=7 ;$ and $>9, n=4)$. Bar graphs indicate mean \pm S.E of SERCA. Western blots were also probed for actin as a loading control

${ }^{*} p<0.05,{ }^{* *} p<0.01$ vs. control group (healthy individuals). 
SERCA protein levels are high in this tissue [6]. Then, we evaluated SERCA protein expression levels in platelets of apparently healthy people and T2DM patients. Our data show that in platelets of people with uncontrolled T2DM, SERCA expression was significantly diminished (mean \pm SEM: $97.11 \pm 2.8$ vs. $25.45 \pm 6.46$, respectively; $p<0.005$ ) (Figure $1 \mathrm{~A}$ ).

$\mathrm{HbA}_{1 c}$ levels are an indicator of different degrees of uncontrolled T2DM [9]. The T2DM Mexican patients were divided according to $\mathrm{HbA}_{1 \mathrm{c}}$ haemoglobin percentage, in order to determine if SERCA expression levels in platelets were disturbed in a manner that correlated with $\mathrm{HbA}_{1 \mathrm{c}}$ [4]. The results confirmed that there is a decrease in SERCA expression levels that depends on $\mathrm{HbA}_{1 \mathrm{c}}$ increase (mean \pm SEM: $89.28 \pm 6.78,52.65 \pm 8.15$, $19.67 \pm 6.29$, to $<5.7,5.7-6.9$, and 7-7.9, respectively; $p<0.005)$ and that, from levels of $\mathrm{HbA}_{1 \mathrm{c}}$ $>8 \%$, SERCA expression levels are undetectable (Figure $1 \mathrm{~B}$ ).

Different scientific reports have established the relevance of SERCA expression disturbances in the genesis of ERS and insulin resistance $[4,5]$. In the same context, we carried out this work because few reports in the literature address the alterations in SERCA expression in T2DM patients [10]. To begin our study, we decided to compare platelets of apparently healthy people and T2DM patients, who had $\mathrm{HbA}_{1 \mathrm{c}}$ between $7 \%$ and $7.9 \%$. The result of this experiment shows that the levels of SERCA expression are diminished in T2DM patients as well as during the state of insulin resistance [5]. On the other hand, we also wanted to assess if at different degrees of uncontrolled T2DM (defined by $\mathrm{HbA}_{1 \mathrm{c}}>7 \%$ ) SERCA expressed disturbances. Finding that there is a progressive decrease in SERCA levels until it disappears, inversely correlated with haemoglobin levels. We believe that SERCA levels can become so low that they are undetectable by the antibody.

In conclusion, as was demonstrated in previous reports, the SERCA expression decrease is not only involved in the genesis of insulin resistance [4, 5]; in fact, once the T2DM is established, SERCA levels decrease even more, depending on the degree of disease control. Moreover, there are solid data proving that overexpression of SERCA in cell culture decreases insulin resistance [4]; likewise, another study showed in uncontrolled T2DM patients that rosiglitazone administration increased SERCA protein expression levels and decreased the insulin resistance [10]. The foregoing centralises the increase in the expression of SERCA as a probable therapeutic strategy.

\section{Conflict of interest}

The authors declare no conflict of interest.

\section{References}

1. Guerrero-Hernández A, Leon-Aparicio D, Chavez-Reyes J, Olivares-Reyes JA, DeJesus S. Endoplasmic reticulum stress in insulin resistance and diabetes. Cell Calcium 2014; 56: 311-22.

2. Ozcan U, Cao Q, Yilmaz E, et al. Endoplasmic reticulum stress links obesity, insulin action, and type 2 diabetes. Science 2004; 306: 457-61.

3. Tian T, Zhao Y, Nakajima S, et al. Cytoprotective roles of ERK and Akt in endoplasmic reticulum stress triggered by subtilase cytotoxin. Biochem Biophys Res Commun 2011; 410: 852-8.

4. Gustavo Vazquez-Jimenez J, Chavez-Reyes J, RomeroGarcia T, et al. Palmitic acid but not palmitoleic acid induces insulin resistance in a human endothelial cell line by decreasing SERCA pump expression. Cell Signal 2016; 28: 53-9.

5. Park SW, Zhou Y, Lee J, Lee J, Ozcan U. Sarco(endo) plasmic reticulum $\mathrm{Ca2+-ATPase} 2 \mathrm{~b}$ is a major regulator of endoplasmic reticulum stress and glucose homeostasis in obesity. Proc Natl Acad Sci USA 2010; 107: 19320-5.

6. Shah RD, Gonzales F, Golez E, et al. The antidiabetic agent rosiglitazone upregulates SERCA2 and enhances TNF-alpha and LPS-induced NF-kappaB dependent transcription and TNF-alpha induced IL-6 secretion in ventricular myocytes. Cell Physiol Biochem 2005; 15: 41-50.

7. Pienimaeki-Roemer A, Kuhlmann K, Böttcher A, et al. Lipidomic and proteomic characterization of platelet extracellular vesicle subfractions from senescent platelets. Transfusion 2015; 55: 507-21.

8. Okutucu B, Dınçer A, Habib Ö, Zıhnıoglu F. Comparison of five methods for determination of total plasma protein concentration. J Biochem Biophys Methods 2007; 70: 709-11.

9. Barbarash O, Gruzdeva O, Uchasova E, Belik E, Dyleva Y, Karetnikova V. Biochemical markers of type 2 diabetes as a late complication of myocardial infarction: a case-control study. Arch Med Sci 2017; 13: 311-20.

10. Randriamboavonjy V, Pistrosch F, Bölck B, et al. Platelet sarcoplasmic endoplasmic reticulum Ca2+-ATPase and mu-calpain activity are altered in type 2 diabetes mellitus and restored by rosiglitazone. Circulation 2008; 117 : 52-60. 